\title{
Des étudiants, touristes chez eux? Du patrimoine urbain en classe de sciences humaines
}

Marie-Claude Larouche

\section{HRONIQUE • Formation des maitres \\ Introduction}

La localisation de Trois-Rivières, au carrefour de voies fluviales stratégiques pour le développement du Québec, fait en sorte que sont rassemblées, en un périmètre assez restreint, nombre de traces intéressantes au regard des réalités historiques et géographiques nationales qui doivent faire l'objet d'un enseignement au primaire. En outre, de nombreux éléments de son cadre urbain font partie d'un patrimoine culturel reconnu d'importance, selon diverses instances officielles.

À nos yeux de formatrice, le microcosme trifluvien représente un environnement exceptionnel pour former les étudiants à l'enseignement des sciences humaines et les sensibiliser à leur futur rôle de passeur culturel. Au fil des ans, nous en avons fait une ressource pour la formation didactique et tiré divers constats. De fait, l'exploitation de ce patrimoine provoque un regain d'intérêt tout à la fois pour les réalités sociales et territoriales à faire étudier par les élèves, et les façons d'engager une démarche active des élèves dans l'étude des traces du passé. Cependant, elle permet aussi de constater une certaine confusion conceptuelle chez les étudiants, lorsqu'on leur demande d'expliciter leur compréhension de l'histoire et de la géographie du Québec...

\section{Le dispositif élaboré : 400 ans d'histoire en deux heures et demie de marche!}

On pourrait dire aussi : 400 ans d'histoire en un kilomètre carré! Après un premier questionnement en classe sur les traces que l'on peut trouver à Trois-Rivières de l'histoire du Québec et sur la notion de patrimoine urbain, nos étudiants effectuent un rallye dans les quartiers centraux 
jouxtant le fleuve et la rivière Saint-Maurice. Ils ont en main un guide de parcours qui mobilise plusieurs techniques liées à l'histoire et à la géographie telles que la lecture de cartes et d'une boussole, l'utilisation de repères chronologiques et l'interprétation de documents iconographiques. En leur proposant ensuite d'analyser les traces observées à la lumière des réalités sociales et territoriales qui figurent dans le programme d'univers social, nous les convions à organiser leurs connaissances sur le site Ligne du temps ${ }^{1}$, en signalant des faits ou des événements significatifs de l'histoire du Québec dont on trouve une trace à Trois-Rivières. Et les exemples ne manquent pas...

\section{Des campements autochtones à la désindustrialisation de la ville}

Si Trois-Rivières est reconnue comme la plus ancienne des villes françaises en Amérique après Québec, on y évoque aussi les campements autochtones où s'établit un poste de traite des fourrures en 1634. Les guerres iroquoises, l'installation de la communauté des Ursulines, les découvertes des frères de La Vérendrye font partie des faits évoqués pour la période de la Nouvelle-France. Différents acteurs importants de l'histoire de Trois-Rivières et du Québec, notamment Pierre Boucher, gouverneur de Trois-Rivières, Ludger Duvernay, journaliste et créateur de la Société Saint-Jean-Baptiste, et Ezekiel Hart, premier député juif élu au parlement, l'ancien premier ministre Maurice Duplessis font l'objet d'étapes dans le rallye. Dans l'un des plus vieux cimetières au pays sont enterrés les restes d'un soldat britannique ayant combattu sur les plaines d'Abraham. Prenant son essor vers 1820, le commerce du bois se constate à l'installation des Baptist, une famille d'entrepreneurs d'origine écossaise qui s'active dans le domaine des scieries et du transport du bois. L'industrie des pâtes et papiers qui s'installe aux abords des cours d'eau vers 1920 entraîne l'urbanisation ultrarapide de la ville, dont la population triple en 30 ans. Les triplex construits à la hâte dans la paroisse Sainte-Cécile tout à côté de la Canadian International Paper (CIP) suffisent alors à peine à loger les travailleurs (et leurs animaux!) venus des campagnes. Devenue glorieusement «capitale mondiale des pâtes et papiers » dans les années 1930, Trois-Rivières vit au tournant $\mathrm{du} 21^{\mathrm{e}}$ siècle une désindustrialisation rapide... On découvre ainsi ce qu'il reste de la CIP, un terrain vague où tout a été rasé sauf un ancien bâtiment transformé depuis en musée (Boréalis, centre d'histoire de l'industrie papetière). Le phénomène religieux n'est pas en reste. De la création à la désaffectation des églises, en passant par leur changement de vocation, en restaurant ou en salle de spectacle, plusieurs traces permettent de l'aborder et d'ouvrir une réflexion sur un changement important suscité par la Révolution tranquille.

\section{Une meilleure compréhension du rôle de passeur culturel... mais une certaine confusion conceptuelle!}

À la suite d'une étude menée auprès de deux groupes d'étudiants à l'automne 2013, au moyen de questionnaires pré et post expérience et d'entretiens de groupe (Larouche, 2016), il appert que l'expérience immersive combinée à la réalisation de la frise chronologique contribue, selon les déclarations des étudiants, à accroître, bien que légèrement, leur familiarisation à l'égard de l'histoire du Québec et des contenus historiques et géographiques du Programme de formation en univers social (Ministère de l'Éducation du Québec, 2001). Chez plusieurs d'entre eux, l'expérience favorise une prise de conscience à l'égard du rôle de passeur culturel en sciences humaines, comme une invitation à établir des liens d'intelligibilité entre divers phénomènes historiques et géographiques plutôt qu'à mémoriser des faits. 
Cependant lors des entretiens avec quelques étudiants, nous avons constaté des difficultés notables à recourir aux concepts explicatifs des réalités sociales pour caractériser les phénomènes historiques et géographiques en jeu. Or, l'exercice même du rôle de passeur culturel n'en dépend-il pas? Nous nous interrogeons ainsi sur les savoirs disciplinaires que possèdent les étudiants et sur leur future capacité à soutenir le raisonnement de leurs élèves selon les perspectives de l'espace et du temps, comme le prescrit le programme. Nous ne pouvons cependant comparer les résultats obtenus avec d'autres travaux, la compétence professionnelle numéro un des enseignants relative à la culture ayant été peu étudiée jusqu’à présent de façon générale ${ }^{2}$, et encore moins dans les sciences humaines.

Comme le signale une étudiante, « c'est plus les touristes qui vont faire un travail de recherche, qui vont aller lire sur les pancartes puis les affiches... C'est dommage, mais je pense que c'est peut-être un legs qu'on peut léguer en tant qu'enseignante, justement d'intéresser les enfants à leur patrimoine urbain ». Relevant du patrimoine culturel, il représente en effet « un actif dont nous commençons à peine, comme citoyens et collectivité, à tirer profit pour l'avenir ", pour reprendre les mots de Roland Arpin (Groupeconseil sur la Politique du patrimoine culturel du Québec, 2000, $4^{\text {e }}$ de couverture). Son exploitation en classe de sciences humaines permet notamment de mesurer l'ampleur de la tâche à accomplir pour former le plus adéquatement possible ces généralistes du primaire, alors que « l'univers social » ne fera pas nécessairement partie de leurs priorités d'enseignement...

\section{Notes}

1 www.lignedutemps.qc.ca

2 Voir Simard, D. (2010). La réforme de l'éducation au Québec : un trésor était caché dedans. Dans M. Mellouki (dir.), Promesses et ratés de la réforme de l'éducation au Québec (p. 75-102). Québec, QC : Presses de l'Université Laval; et Simard, D. et Falardeau, É. (2012). Regards d'enseignants sur le caractère culturel de leur profession. Dans M. Giglio et S. Boéchat-Heer (dir.), Innovations et réformes éducatives (p. 15-30). Bienne : HEP-BEJUNE.

\section{Références}

Groupe-conseil sur la Politique du patrimoine culturel du Québec (GPPCQ). (2000). Notre patrimoine, un présent du passé. Repéré à http://bel.uqtr.ca/1239/1/6-19-681-20060117-1.pdf

Larouche, M.-C. (2010). Guide de parcours «Quelques pas dans l'histoire et le patrimoine du Québec ». Document PDF inédit.

Larouche, M.-C. (2016). L'exploitation pédagogique du patrimoine urbain de Trois-Rivières : quel apport à la formation didactique en sciences humaines?. Dans M.-C. Larouche, J. Burgess et N. Beaudry (dir.), Éveil et enracinement: Approches pédagogiques innovantes du patrimoine culturel (p. 95-115). Québec, QC : Presses de l'Université du Québec.

Ministère de l'Éducation du Québec (MEQ). (1997). L'école, tout un programme. Énoncé de politique éducative. Repéré à http:// srp.csrs.qc.ca/evaluation/Documents/Lecole tout un programme.pdf

Ministère de l'Éducation du Québec (MEQ). (2001). Programme de formation de l'école québécoise, éducation préscolaire, enseignement primaire. Québec, QC : Gouvernement du Québec.

\section{Pour citer cet article}

Larouche, M-C. (2017). Des étudiants, touristes chez eux ? Du patrimoine urbain en classe de sciences humaines. Formation et profession, 25(2), 102-104. http://dx.doi.org/10.18162/fp.2017.a126 